\title{
Effect of temperature on rate of left ventricular pressure fall in humans
}

\author{
RICHARD A LUKE, * CHARLES E GILLBE, $\dagger$ ROBERT S BONSER, $\ddagger$ \\ MATTHIAS PANETH, $\dagger$ DAWN SOMERSET, * JUDITH THOMAS, $\ddagger$ \\ DEREK G GIBSON *
}

From the Departments of ${ }^{\star}$ Cardiology, $\uparrow$ Anaesthetics, and $\ddagger$ Cardiac Surgery, Brompton Hospital, London

SUMMARY The time constant $(T)$ of left ventricular pressure fall is widely used as an index of ventricular "relaxation". It is not known whether its rate limiting step is deactivation, an enzymię energy consuming reaction whose rate is therefore sensitive to temperature, or elastic recoil. Tid distinguish between these possibilities, the time constant was measured by simple logarithmi ( $(\mathrm{log}$ ) and exponential (Texp) methods in 12 patients during cooling before coronary artery grafting. Ventricular loading conditions were altered by transfusion from bypass to maintain arterial and left atrial pressures constant in individual patients, though heart rate fell from $86(8.4)$ to $68(10)$ beats $/ \mathrm{min}$. Tlog increased from $49(10) \mathrm{ms}$ mean (SD), at $37^{\circ} \mathrm{C}$ to $86(15) \mathrm{ms}$ at $31^{\circ} \mathrm{C}$, and Texp from $63(14)$ at $37^{\circ} \mathrm{C}$ to $112(23) \mathrm{ms}$ at $31^{\circ} \mathrm{C}$ with intermediate values at $34^{\circ} \mathrm{C}$. Texp prove ${ }^{\circ}$ sensitive to "noise" at low temperatures, but the overall change in Tlog with temperature was $9 \%$ per ${ }^{\circ} \mathrm{C}$-considerably less than that observed experimentally for the rate of tension decline of isolated myocardium, and possibly itself an overestimate because of the concomitant fall in hear rate.

The relatively small effect of temperature on Tlog in humans, associated with a considerable loa里 sensitivity appearing under hypothermic conditions, does not favour simple dependence or deactivation as the rate limiting step of left ventricular pressure fall, but suggests that its determinants may be complex.

Though the time constant $(T)$ of left ventricular pressure fall during isovolumic relaxation is widely used as an index of ventricular relaxation and of diastolic function, ${ }^{1-3}$ underlying mechanisms in humans have not been defined in detail. Since it depends on events occurring before mitral valve opening, it is not directly affected by left ventricular filling, and is thus likely to reflect either the rate of termination of the active state within the myocardium - that is deactivation-or elastic restoring forces, possibly accompanied by a change in left ventricular cavity shape. Ventricular deactivation is a process requiring energy and so its rate is temperature sensitive. ${ }^{45}$ When cardiopulmonary bypass is initiated before coronary artery surgery the heart is cooled; this provides an opportunity to assess the effect of temperature on early diastole. We therefore

Requests for reprints to Dr Derek G Gibson, Cardiac Department, Brompton Hospital, London SW3 HP.

Accepted for publication 10 January 1989 aimed to determine how myocardial temperature changes affected the time course of left ventriculas pressure fall to help to understand clinical disturban ces seen in humans.

\section{Patients and methods}

We studied eleven men and one woman (aged 42-65로. undergoing coronary artery surgery for relief of angina pectoris. We excluded those with unstablê angina, clinically significant left main stem stenosiso associated valve disease, or impaired left ventriculaf function (ejection fraction $<50 \%$ ). The resting preoperative electrocardiogram was normal in eighe and showed inferior $Q$ waves in four. At operation between two and five grafts were attached. The protocol was approved by the ethics committee of the National Heart and Chest Hospital, with thథ requirement that data collection should be complete within 30 minutes. All patients gave witnessed infor $\cong$ med consent and there were no complications. 
ANAESTHETIC TECHNIQUE

All patients were premedicated with papaveretum $10-20 \mathrm{mg}$ and hyoscine $0.2-0.4 \mathrm{mg}$. The left radial artery was cannulated under local anaesthesia and arterial pressure was monitored continuously before and throughout induction. General anaesthesia was induced with fentanyl $8 \mu \mathrm{g} / \mathrm{kg}$. Pancuronium $0 \cdot 1-$ $0.15 \mathrm{mg} / \mathrm{kg}$ was administered to obtain neuromuscular blockade. Anaesthesia was maintained by intermittent positive pressure ventilation with air, oxygen, and isoflurane $1-2 \%$.

\section{SURGICAL TECHNIQUE}

After sternotomy, cardiopulmonary bypass was established with right atrial drainage and return to the ascending aorta. A $5 \mathrm{~F}$ Millar catheter with pressure tip transducer (Millar Instruments model SPC-350, with a temperature coefficient of less than $\pm 1 \mathrm{~mm} \mathrm{Hg}$ between 23 and $38^{\circ} \mathrm{C}$ ) was then introduced into the left ventricle through an apical vent incision. A 16 gauge cannula was inserted into the left atrium and connected to a fluid filled pressure transducer. We used this to monitor the left atrial pressure. Myocardial temperature was measured by a temperature probe (YSI series 500 , no 586 ) inserted into the posterior left ventricular wall. In the first five patients, the septal temperature was also monitored with a hypodermic probe (YSI, series 500, no 513, 20 gauge). Because it was identical with that of the posterior wall within five minutes of a change in temperature in all these cases we did not continue to measure the temperature of the septum in subsequent patients. The heart was rewarmed if neces- sary to the normothermic range $\left(36.5\right.$ to $\left.37.5^{\circ} \mathrm{C}\right)$ and bypass discontinued with a mean left atrial pressure of $2-6 \mathrm{~mm} \mathrm{Hg}$, or higher if arterial pressure appeared inadequate. After five minutes of equilibrium, left ventricular pressure was recorded and simultaneous mean left atrial pressure and myocardial temperature were noted. The filling pressure was then increased by transfusion from the bypass up to a maximum of $15 \mathrm{~mm} \mathrm{Hg}$ in order to increase arterial pressure by $20 \%$ or more. A second set of recordings was obtained after one minute. Bypass was then resumed, and the heart was cooled to the hypothermic range (31 to $32.5^{\circ} \mathrm{C}$ ), and further recordings were obtained at two filling pressures as for the normothermic range. An additional set of recordings was made at an intermediate temperature when possible within the time constraints of the protocol. There was no electrocardiographic evidence of left ventricular ischaemia at any time during the study.

\section{CALIBRATION AND RECORDINGS}

The Millar catheter was immersed in saline for at least $\mathbf{2 0}$ minutes before use to minimise zero drift and was then calibrated electrically. Zero pressure was taken as atmospheric. At the end of the study, drift was less than $2 \mathrm{~mm} \mathrm{Hg}$ for all patients. Left ventricular pressure was recorded on tape and a hard copy obtained by a photographic recorder working at effective paper speeds of 50 and $200 \mathrm{~mm} / \mathrm{s}$ (Meddars model 1100 with LS-8 recorder, Honeywell, fig 1). Zero left atrial pressure was taken at the mid-atrial level. Temperature probes were individually precalibrated.
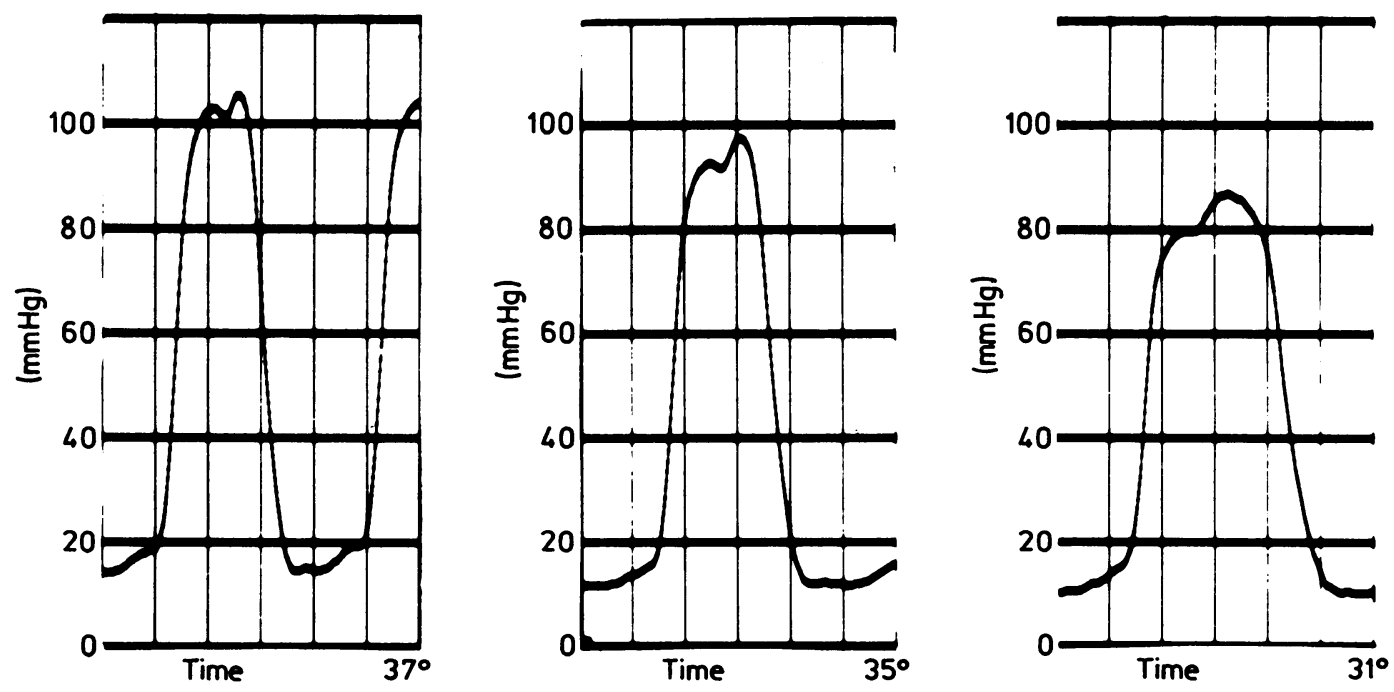

Fig 1 Effect of temperature on the left ventricular pulse at $37^{\circ} \mathrm{C}$ (left panel), $35^{\circ} \mathrm{C}$ (centre panel), and $31^{\circ} \mathrm{C}$ (right panel). 
STATISTICAL ANALYSIS

Because values of $T$ might have been affected by varying loading conditions, arterial and left atrial pressures were matched as closely as possible at different temperature levels within individual patients. Left ventricular pressure recordings were digitised every $5 \mathrm{~ms}$. We took isovolumic relaxation as the interval from the time of peak negative $\mathrm{dP} / \mathrm{dt}$ until ventricular pressure was $5 \mathrm{~mm} \mathrm{Hg}$ above the end diastolic pressure of the preceding beat, and analysed data points only during this period. We determined peak left ventricular and mean left atrial pressures directly from the appropriate recordings.

We calculated the time constant of relaxation (Tlog) by the semilogarithmic method originally suggested by Weiss et al, ${ }^{6}$ according to the function $\mathbf{P}=\mathbf{P o e}-\frac{\mathbf{t}}{\mathbf{T}}$, where Po is pressure at zero time-

that is simultaneous with $\mathrm{dP} / \mathrm{dt}-\mathbf{P}$ the pressure at any time $(t)$ after this, and $T$ the time constant of pressure fall. This derivation assumes that the asymptote $(\mathrm{Pb})$ of pressure fall is zero, and is measured from a plot of the natural logarithm of pressure against time. For all recordings, the correlation coefficient of this plot, using a linear fit, was greater than 0.98 . We also calculated the time constant from the plot of negative $\mathrm{dP} / \mathrm{dt}$ against $P$. This method gives $T$ as the slope of the line, and the asymptote is derived from the intercept. ${ }^{7}$ When pressure fall was prolonged at low temperatures, $\mathrm{dP} /$ dt was subject to "noise"; and we did not use values whose correlation coefficient fell below 0.90 in subsequent analysis.

We used average values for two beats and, for the purpose of analysis, the three temperature ranges defined in the experimental protocol were considered: normothermic (above $36^{\circ} \mathrm{C}$ ), intermediate $\left(33-36^{\circ} \mathrm{C}\right)$, and hypothermic $\left(<33^{\circ} \mathrm{C}\right)$.

Preoperative left ventricular angiograms were digitised frame by frame $^{8}$ to determine ejection fraction and regional wall motion in nine patients. In three patients angiograms were technically unsuitable for such analysis because of extrasystoles but left ventricular function seemed to be unimpaired on visual assessment.

\section{Results}

\section{HAEMODYNAMIC VARIABLES}

Heart rate fell with temperature from a mean value of $86(8.4)$ beats per minute in the range $35-37^{\circ} \mathrm{C}$ to 77 $(9.5)$ beats per minute in the intermediate temperature range, and $68(10)$ beats per minute at the lowest range, between 31 and $33^{\circ} \mathrm{C}$. The relation between heart rate and temperature was consistent, and given by the regresssion equation:

Heart rate $=3.78$ (temperature) -55 beats $/ \min _{,}$, with a correlation coefficient of $0.69(p<0.001)$, an a standard error of the estimate of 9.8 beats $/ \mathrm{min}$.

At normal temperature, peak arterial systolic pres sure was 105 (15) $\mathrm{mm} \mathrm{Hg}$ with a mean atrial pressure of 8 (3) $\mathrm{mm} \mathrm{Hg}$, compared with 106 (15) and $\frac{8}{8}$ (4) $\mathrm{mm} \mathrm{Hg}$, respectively, in the intermediate range and $104(15)$ and $9.5(4.5) \mathrm{mm} \mathrm{Hg}$ at the lowest temperatures. There was no significant differences between these values at the three temperature ranges. Negative $\mathrm{dP} / \mathrm{dt}$ depended on both temperature an£ peak systolic pressure, as given by the multipes regression equation:

Peak negative $\mathrm{dP} / \mathrm{dt}=-2550+71$

(temperature) - 10 (systolic pressure), where $r=0.87$ and $p<0.001$ for both predictors. N

TIME CONSTANTS

Above $36^{\circ} \mathrm{C}$.- Mean values of $\mathrm{Tlog}$ and $\mathrm{Texp}$ wer 49 (10) and 63 (14) ms respectively. In patients studied under normothermic conditions, the correlas tion coefficient of the relation between $T l o g$ and heare rate was -0.59 , and that between Texp and heart rate was $-0 \cdot 33$. Neither was statistically significan ( $p<0.10$ and 0.20 , respectively).

Temperature $33-36^{\circ} \mathrm{C}$.- Mean values of $\mathrm{Tlog}$ and Texp increased to $72(11)$ and $87(20)$ respectively these values were significantly longer than those recorded in the normothermic range $(p<0.01)$.

Temperature $<33^{\circ} \mathrm{C}$. - The mean value of Tlog was further prolonged to 86 (15) ms. Because "noise" associated with the trace of $\mathrm{dP} / \mathrm{dt}$ at lower temperatures, only four data pairs could be cap culated for Texp, whose mean value was 112 (23) ms When values obtained under intermediate or hypo? thermic conditions (that is below $36^{\circ} \mathrm{C}$ ) were conssidered, again the correlation between Tlog ang spontaneous heart rate was not significant $(r=-0 \cdot 28)$. Overall, the relation between Tlog and temperature was given by the equation:

$$
\text { Tlog }=330-7 \cdot 7 \text { (temperature), }
$$

with a correlation coefficient of $-0.87(p<0.0010)$ and standard error of the estimate $34 \mathrm{~ms}$ (fig 2N Directionally similar changes were seen in Texp, buff their statistical significance could not be calculated because only four values were available in the low temperature range.

ASYMPTOTE OF PRESSURE FALL
The asymptote of pressure fall can be calculated the pressure at which $\mathrm{dP} / \mathrm{dt}$ becomes zero. The mean value of $\mathrm{Pb}$ at normal temperatures was - 18 (12) $\mathrm{mm} \mathrm{Hg},-9$ (7) at intermediate, and - 5 


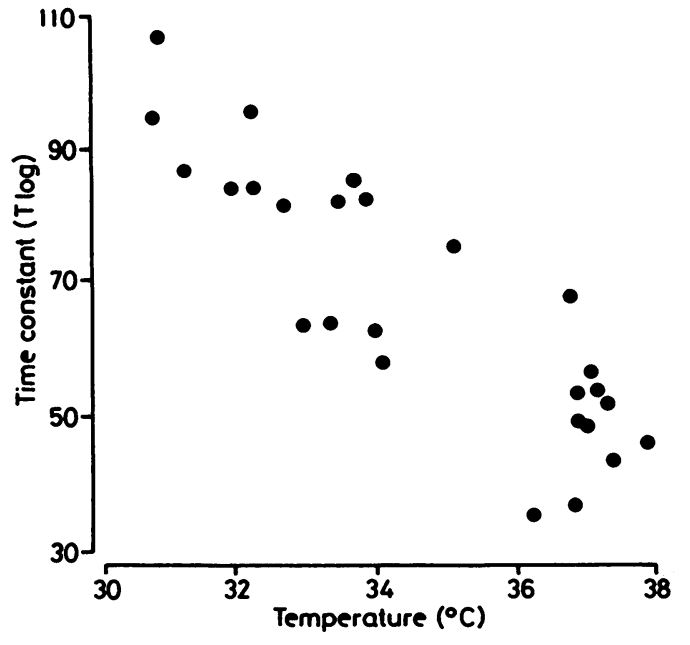

Fig 2 Relation between myocardial temperature and Tlog during myocardial cooling.

(13) $\mathrm{mm} \mathrm{Hg}$ at the lowest temperatures. These values all differed significantly from zero ( $p<0.01$ ), but were unrelated to temperature.

ANALYSIS OF ANGIOGRAMS

Angiograms adequate for analysis were obtained in nine patients. The ejection fraction ranged from $57 \%$ to $84 \%$. Contour displays showed a completely normal pattern of regional wall motion in three. The pattern of "diagonal contours" for the apical or inferior segment was present in five, without any other abnormality in four of these and with inferior hypokinesis in one. In one patient, there was a change in cavity shape during isovolumic relaxation, with early outward motion of the anterior wall, and delayed inward motion along the inferior wall.

\section{Discussion}

Although the time constant ( $T$ ) of relaxation is widely used to assess the left ventricle during diastole in humans, little is known of what determines its length in individual patients. Problems in interpreting it are compounded by inconsistent definitions. In the intact heart, the word "relaxation" has been used to mean deactivation, ${ }^{10}$ outward ventricular wall motion or wall thinning, ${ }^{11}$ and even a return to precontractile configuration. ${ }^{12}$ Not only are these various definitions mutually incompatible, but they include isovolumic relaxation and all three phases of ventricular filling. Even if the mechanism of ventricular pressure fall were completely understood, it would still not be clear which of these various processes the time constant actually assesses.

Measuring a time constant of pressure fall in the intact heart presents obvious technical difficulties. Early experiments showed that tension decayed exponentially in isolated muscle, ${ }^{13}$ and so could be quantified as a rate constant. Weiss et al proposed that Tlog, calculated from a semilogarithmic plot of ventricular pressure, could be used in a similar way to follow relaxation in the intact heart ${ }^{6}$-assuming that the pressure fall was exponential, the ventricle isovolumic, and the asymptote zero. More recent methods for calculating $T$ have shown that the asymptote is not zero, ${ }^{17}$ but is frequently large and negative. There is no theoretical reason why pressure fall should be exponential during isovolumic relaxation, particularly in disease, and striking departures have been noted in hearts damaged by ischaemia or with severe left ventricular hypertrophy. ${ }^{14}$ Our patients had normal or near normal patterns of left ventricular wall motion before operation, and showed no evidence of acute ischaemia at the time that measurements were made. Correlation coefficients for calculation of Tlog were greater than 0.98 throughout the study; this indicates that assuming an exponential was probably not a major source of error. Calculation of Texp at low temperatures was, however, affected by "noise" on the $d P / d t$ trace rather than by any configurational change in left ventricular pressure fall, but this method did allow us to show that values of the asymptote were consistently subatmospheric. It has been suggested that the asymptote may differ from zero simply because pericardial or intrathoracic pressure are sub-atmospheric. ${ }^{15}$ Although the values we obtained when the chest and pericardium were open were less negative than those previously reported at cardiac catheterisation, ${ }^{1}$ they were consistently below zero, suggesting that factors other than pericardial or intrathoracic pressure contribute to the asymptote of left ventricular pressure fall in intact man.

A second problem in calculating $T$ is to define the time of mitral valve opening. We used the simple criterion of taking the time when left ventricular pressure was $5 \mathrm{~mm} \mathrm{Hg}$ above end diastolic pressure. Other criteria have been used, including arbitrary points on the pressure curve, $\mathrm{at}^{3616}$ or above ${ }^{151718}$ the end diastolic pressure of the previous beat, pressure crossover, ${ }^{7}$ or the time of mitral valve opening determined by angiography ${ }^{2}$ or echocardiography. ${ }^{12}$ In patients with ventricular disease, when left atrial pressure is significantly raised, the isovolumic relaxation time may be short or even zero, ${ }^{19}$ which invalidates the basis on which time constant is calculated.

Haemodynamic influences on $T$ have been extensively studied. It is generally agreed that $T$ shortens 
as the heart rate increases, ${ }^{125}$ but the effect of arterial pressure is variable, with some studies reporting a lengthening of $T$ as systolic pressure increases ${ }^{16} 1720$ and others no change. ${ }^{521} 22$ Any influence of filling pressure ${ }^{17}$ is probably indirect and due to its effect on arterial pressure. ${ }^{15}$ The use of different anaesthetic agents has been invoked as a cause of these discordant experimental results. ${ }^{17}$ Isoflurane is known to cause vasodilatation, depress contractility, and possibly to inhibit slow calcium channels ${ }^{23}$; but it seems unlikely that it influenced our results to any significant extent since the level of anaesthesia was maintained constant throughout the procedure. If $T$ does indeed reflect early diastolic processes, therefore, it is likely to be load dependent, ${ }^{124}$ so that in the present study we matched values of arterial and left atrial pressures at different temperatures in individual patients.

The rate limiting step of left ventricular pressure fall in humans is still uncertain. During isovolumic relaxation, it presumably reflects the effects either of deactivation or of elastic restoring forces, though their relative preponderance has not been established. Decay of the active state is associated with calcium uptake into the sarcoplasmic reticulum. Because this is an enzymic process requiring ener$\mathrm{gy},{ }^{45}$ its rate will be very sensitive to temperaturevalues of $17 \%$ per degree centigrade in the rate of tension fall in isolated myocardium have been shown experimentally in the range $28-33^{\circ} \mathrm{C} . .^{25}$ Alternatively, restoring forces ${ }^{26-28}$ residing in elastic elements within the myocardium and energised during the previous systole may determine the time course of left ventricular pressure fall. The effect of temperature on this mechanism would be significantly less than that on an enzymic process, although not absent altogether, because viscosity changes with temperature. ${ }^{29}$

Cooling the myocardium during cardiopulmonary bypass provides an excellent opportunity to investigate these possibilities in humans within a temperature range of approximately $7^{\circ} \mathrm{C}$. Clearly, normal hearts cannot be studied in this way, but we selected patients with normal ventricular function judged in terms of ejection fraction and regional systolic and diastolic wall motion. The range of values of Texp at normal temperatures overlapped those previously reported at cardiac catheterisation in controls ${ }^{18}$; those of $T$ log were a little longer, but this difference was almost certainly related to the less negative value of the asymptote in our patients studied with the chest open. ${ }^{1}$ There was no evidence of acute ischaemia at the time that measurements were made. While minor differences between our results and those that would have been obtained in controls are expected, any residual effects of ischaemia are likely to have become smaller rather than larger as temperature fell, so it seems improbable that there were major discrepancies.

Our results confirm that the time constant of pressure fall was indeed somewhat prolonged withos cooling in humans, amounting to approximately $9 \%$ per degree Centigrade. However, not only was thiso

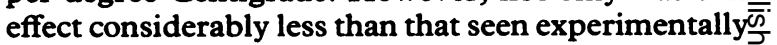
on the rate of tension decline, but, moreover, may actually be an overestimate. There was a consistento fall in heart rate in our patients, which would be expected per se to lengthen $T$, irrespective of any? change in temperature. Although we were able to $\vec{\omega}$ eliminate any overall dependence on heart rate in ouro patients, the possibility of an intrapatient effect remains. From the data of Thompson et al, ${ }^{1}$ this fallo in heart rate would be expected to increase Tlog by $\vec{c}$ approximately $4 \mathrm{~ms}$, or $10 \%$ of the total change between 38 and $32^{\circ} \mathrm{C}$. Decreased catecholamineo secretion, ${ }^{30}$ and any direct effect of temperature on ventricular activation might also have been expectedto prolong $T$ indirectly. Finally, as we have briefly reported elsewhere, ${ }^{31}$ values of $T$ become very sen sitive to arterial and venous pressure at temperatureso below $34^{\circ} \mathrm{C}$. This means that the effect of tem-0 perature itself becomes load dependent, being even further diminished as arterial pressure rises.

We conclude, therefore, that although the time constant of left ventricular pressure fall is prolonged with cooling in humans, the basis of this effect isD complex. Its extent is considerably less than that in the rate of tension decline in isolated myocardium, ${ }^{25}$ although the size of this discrepancy is difficult to quantify because load dependence develops in humans below $34^{\circ} \mathrm{C}$. The results suggest that the rate of deactivation is not the only, or even a dominant $\overline{0}$ cause of the rate of pressure fall in man, and that underlying mechanisms are complex and multiple We feel that it may be unwise to ascribe variation of the time constant of pressure fall in all circumstances to changes in any single entity, particularly one so loosely defined as "relaxation".

RAL was supported by a grant from the New? Zealand Heart Foundation.

\section{References}

1 Thompson DS, Waldron CB, Juul SM, et al Analysis of left ventricular pressure during isovolumic relaxation in coronary artery disease. Circulation? 1982;65:690-7.

2 Fioretti P, Brower RW, Meester GT, Serruys PW Interaction of left ventricular relaxation and filling during early diastole in human subjects. $\mathrm{Am} \mathrm{J}$ Cardio 1980;46:197-203.

3 Katayama J, Kumada T, Fujii T, et al. Clinical charac 
teristics of left ventricular pressure decline during isovolumic relaxation in normal and diseased hearts. Am Heart $J$ 1984;107:332-7.

4 Langer GA. Ion fluxes in cardiac excitation and contraction and their relation to myocardial contractility. Physiol Rev 1968;48:708-75.

5 Nayler WG, Williams A. Relaxation in heart muscle: some morphological and biochemical considerations. Eur Heart $J$ 1978;7(suppl):35-50.

6 Weiss JL, Fredericksen JW, Weisfeldt ML. Hemodynamic determinants of the time-course of fall in canine left ventricular pressure. $J$ Clin Invest 1976;58:751-60.

7 Weisfeldt ML, Weiss JL, Frederiksen JT, Yin FCP. Quantification of incomplete left ventricular relaxation: relationship to the time constant for isovolumic pressure fall. Eur Heart J 1980;1:A119-29.

8 Gibson DG, Prewitt TA, Brown DJ. Analysis of left ventricular wall movement during isovolumic relaxation and its relation to coronary artery disease. $\mathrm{Br}$ Heart J 1976;38:1010-9.

9 Gibson DG, Greenbaum RA, Pridie RB, Yacoub MH. Correction of left ventricular asynchrony by coronary artery surgery. Br Heart $J$ 1988;59:304-8.

10 Grossman W, McLaurin LP. Diastolic properties of the left ventricle. Ann Intern Med 1976;84:316-26.

11 Friedman MJ, Sahn DJ, Burris HA, Allen HD, Goldberg SJ. Computerized echocardiographic analysis to detect abnormal systolic and diastolic left ventricular function in children with aortic stenosis. $\mathrm{Am} \mathrm{J} \mathrm{Cardiol}$ $1979 ; 44: 478-86$.

12 Brutsaert DL, Rademakers FE, Sys SU, Gillebert TC. Housmans PR. Analysis of relaxation in the evaluation of ventricular function of the heart. Prog Cardiovasc Dis 1985;28:143-63.

13 Parmley WW, Sonnenblick EH. Relation between mechanics of contraction and relaxation in mammalian cardiac muscle. Am J Physiol 1969;216: 1084-91.

14 Rousseau MF, Verher C, Detry J-M, Brasseur L, Pouleur H. Impaired early left ventricular relaxation in coronary artery disease: effects of intracoronary nifedipine. Circulation 1980;62:764-72.

15 Gaasch WH, Carroll JD, Blaustein AS, Bing OHL. Myocardial relaxation: effects of preload on the time course of isovolumetric relaxation. Circulation 1986;73:1037-41.

16 Karliner JS, LeWinter MM, Mahler F, Engler R, O'Rourke RA. Pharmacologic and hemodynamic influences on the rate of isovolumic left ventricular relaxation in the normal conscious dog. J Clin Invest
1977;60:511-21.

17 Raff GL, Glantz SA. Volume loading slows left ventricular relaxation rate: evidence of load dependent relaxation in the dog heart. Circ Res 1981;48:813-24.

18 Carroll JD, Hess OM, Hirzel HO, Krayenbuehl HP. Dynamics of left ventricular filling at rest and during. exercise. Circulation 1983;68:59-67.

19 Mattheos M, Shapiro E, Oldershaw PJ, Sacchetti R, Gibson DG. Non-invasive assessment of changes in left ventricular relaxation by combined phono-, echo-, and mechanocardiography. $\mathrm{Br}$ Heart $J$ $1982 ; 47: 253-60$.

20 Blaustein AS, Gaasch WH. Myocardial relaxation 6. Effects of beta-adrenergic tone and asynchrony on LV relaxation rate. Am J Physiol (H) 1983;244:417-22.

21 Frederiksen JW, Weiiss JL, Weisfeldt ML. Time constant of isovolumic pressure fall: determinants in the working left ventricle. Am J Physiol (H) 1978;235:701-6.

22 Eichhorn P, Grimm J, Koch R, Hess O, Carroll J, Krayenbuehl HP. Left ventricular relaxation in patients with left ventricular hypertrophy secondary to aortic valve disease. Circulation 1982;65:1395-404.

23 Eger EI II. Isoflurane: a review. Anesthesiology 1981;55:559-76.

24 Brutsaert DL, Housmans PR, Goethals MA. Dual control of relaxation: its role in the ventricular function in the mammalian heart. Circ Res 1974;44:637-52.

25 Zile MR, Gaasch WH, Wiegner AW, Robinson KG, Bing OHL. Mechanical determinants of maximum isotonic lengthening rate in rat left ventricular myocardium. Circ Res 1987;60:815-23.

26 Katz LN. The role played by the relaxation in filling of the ventricle. Am J Physiol 1930;95:542-50.

27 Winegrad S, Weisberg A, McClennan G. Are restoring forces important to relaxation? Eur Heart J 1980;1:suppl A:59-66.

28 Sonnenblick EH. The structural basis and important of restoring forces and elastic recoil for the filling of the heart. Eur Heart J 1980;1:A-107-10.

29 Templeton GH, Wildenthal K, Willerson JT, Reardon WC. Influence of temperature on the mechanical properties of cardiac muscle. Circ Res 1974;34: 624-34.

30 Goldberg LI. Effects of hypothermia on contractility of the intact dog heart. Am J Physiol 1958:92-8.

31 Luke RA, Gillbe CE, Bonser RS, Paneth M, Gibson DG. Effect of temperature on the rate of left ventricular pressure fall in humans [Abstract]. Circulation 1987;76(suppl IV):512. 